\title{
Herb-induced cardiotoxicity from accidental aconitine overdose
}

\author{
Sujata $\underline{\text { Sheth }}{ }^{1}$, BSc, MD, Elaine Ching Ching $\underline{\operatorname{Tan}^{1}}$, MRCS, FAMS, Hock Heng $\underline{\operatorname{Tan}^{2}}$, FRCS, DABT, Leslie $\underline{\operatorname{Tay}^{3}}$, FAMS, FRCP
}

\begin{abstract}
Patients who overdose on aconite can present with life-threatening ventricular arrhythmia. Aconite must be prepared and used with caution to avoid cardiotoxic effects that can be fatal. We herein describe a case of a patient who had an accidental aconite overdose but survived with no lasting effects. The patient had prepared Chinese herbal medication to treat his pain, which resulted in an accidental overdose of aconite with cardiotoxic and neurotoxic effects. The patient had ventricular tachycardia, bidirectional ventricular tachycardia and ventricular fibrillation. Following treatment with anti-arrhythmic medications, defibrillation and cardiopulmonary resuscitation, he made an uneventful recovery, with no further cardiac arrhythmias reported.
\end{abstract}

Keywords: aconite, aconitine herb, bidirectional ventricular tachycardia, overdose, cardiotoxic

\section{INTRODUCTION}

Aconitum plant species, also known as aconite, contain aconitine and other related alkaloids that are potent cardiotoxins and neurotoxins. ${ }^{(1)}$ Aconite can be found in Europe, North America and Asia. Aconite poisoning occurs when the wild plant is eaten by mistake or in the form of medicinal preparations such as processed roots, tinctures, pastes, tablets or proprietary capsules. Aconite root is used in traditional Chinese medicine for pain relief. ${ }^{(1)}$ However, the root tubers are very toxic and must be prepared carefully. We herein present a case of aconite poisoning from the consumption of a herbal broth in which the patient presented with life-threatening arrhythmia and cardiovascular collapse.

\section{CASE REPORT}

A 34-year-old man presented to the emergency department, Changi General Hospital, Singapore, at 2.44 am with the chief complaint of numbness all over his body, weakness and shortness of breath. At presentation, his vital signs were: blood pressure $64 / 43 \mathrm{mmHg}$, pulse rate $185 \mathrm{bpm}$, respiratory rate 24/minute, temperature $36.3^{\circ} \mathrm{C}$ and oxygen saturation $99 \%$ on $2 \mathrm{~L}$ oxygen. His hypocount was $6.3 \mathrm{mmol} / \mathrm{L}$. He was lethargic but arousable to painful and verbal stimuli, and was oriented to person and place. The patient's respiratory sounds were equal bilaterally, with warm extremities, and there were no lateralising signs neurologically.

Initial electrocardiography (ECG) (Fig. 1) showed a pattern of fascicular ventricular tachycardia (VT). Intravenous (IV) adenosine (6 mg followed by $12 \mathrm{mg}$ ) administered in initial attempts to rule out supraventricular arrhythmia did not result in any change to his ECG. The toxicology service that was consulted suspected aconitine poisoning based on the patient's clinical presentation. We were advised to start the patient on IV amiodarone $150 \mathrm{mg}$ and $50 \mathrm{~g}$ of activated charcoal orally. After amiodarone was administered, his blood pressure was 167/112, pulse rate 154 bpm, respiratory rate 24 /minute and oxygen saturation was $100 \%$ on room air. The patient was more awake and alert at this point, and could tolerate the charcoal. Repeat ECG at 3.46 am showed frequent premature ventricular contractions (PVCs) with fascicular bigeminy (Fig. 2).

On further history-taking, it was discovered that the patient had a history of ankylosing spondylitis. He had found a recipe for pain alleviation involving traditional Chinese herbs on the Internet, and bought the herbs and roots from a Chinese medicinal store. Following the instructions, he brewed the medicinal broth for two hours and subsequently drank about $200 \mathrm{~mL}$ of it. Twenty minutes after drinking, the patient felt his limbs swelling up and numbness spread throughout his body. He felt giddy but did not have palpitations, chest pain or abdominal pain. The patient was conveyed to the hospital. The family brought the handwritten recipe as well as samples of the herbs to the emergency department. The recipe contained 13 ingredients, including $30 \mathrm{~g}$ of chuanwu and $30 \mathrm{~g}$ of caowu.

At $4.01 \mathrm{am}$, an additional ECG was done, which showed bidirectional VT (Fig. 3). Cardiology was consulted and an additional dose of IV amiodarone $150 \mathrm{mg}$ was given at $4.10 \mathrm{am}$. The patient was then admitted to the intensive care unit (ICU). While waiting for a bed, he became unresponsive at $4.26 \mathrm{am}$, and had a VT rhythm with a pulse. Synchronised cardioversion was performed at $200 \mathrm{~J}$. The patient's heart rhythm became VT with no pulse at 4.27 am, when $200 \mathrm{~J}$ of direct current (DC) shock was delivered and cardiopulmonary resuscitation (CPR) was initiated. At 4.29 am, ventricular fibrillation was observed. Hence, another DC shock of $200 \mathrm{~J}$ was delivered and CPR resumed. Thereafter, the patient's rhythm demonstrated ventricular bigeminy with the following vital signs: blood pressure 133/86 mmHg, pulse rate $120 \mathrm{bpm}$ and oxygen saturation of $98 \%$ on room air. The patient was

${ }^{1}$ Accident and Emergency Department, ${ }^{2}$ Clinical Toxicology Consultation Service, Accident and Emergency Department, ${ }^{3}$ Cardiology Department, Changi General Hospital, Singapore Correspondence: Dr Sujata Kirtikant Sheth, Consultant, Accident and Emergency Department, Changi General Hospital, 2 Simei Street 3 , Singapore 529889. sujata_sheth@cgh.com.sg 


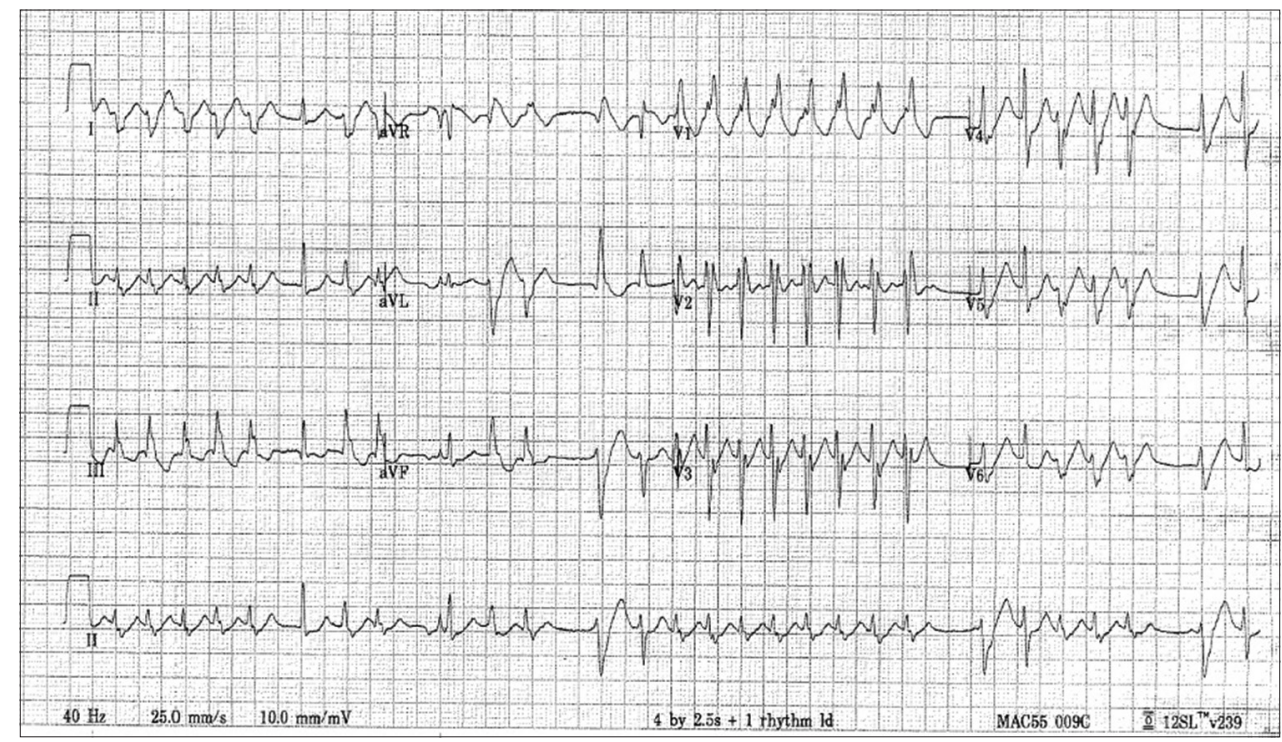

Fig. 1 ECG shows fascicular ventricular tachycardia.

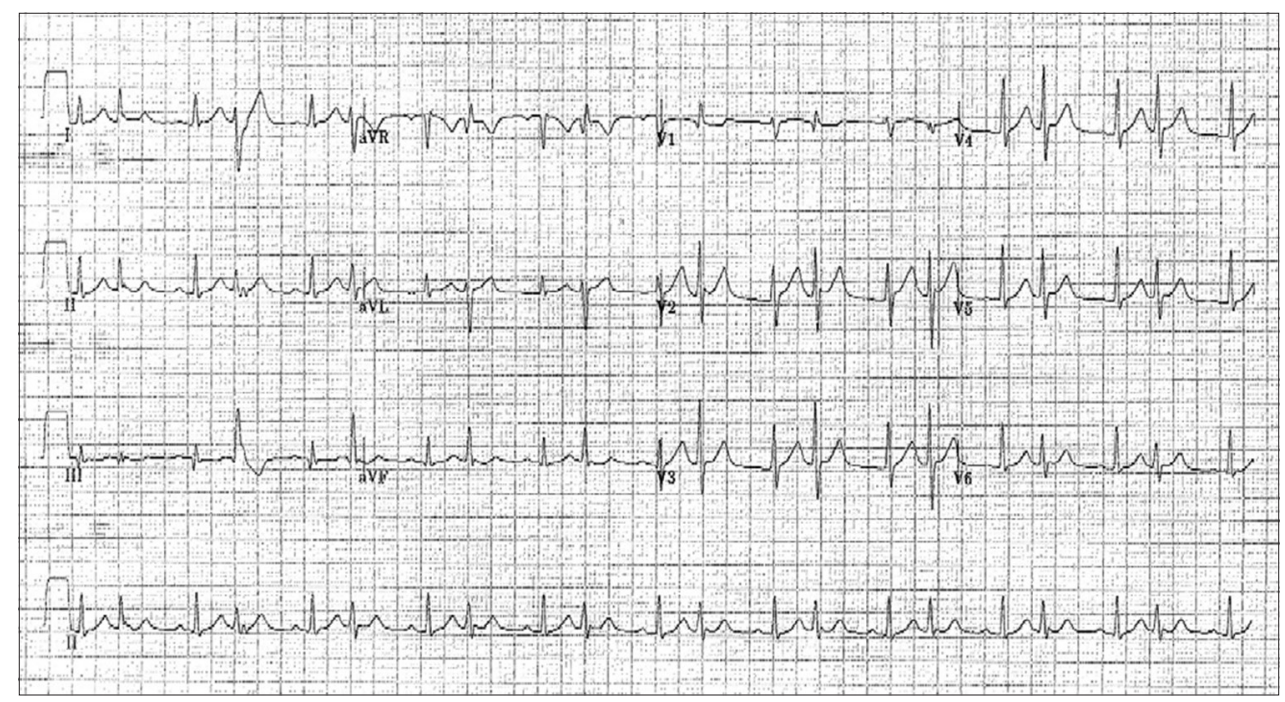

Fig. 2 ECG shows fascicular bigeminy.

intubated and sent to the ICU at $5.50 \mathrm{am}$, where he was stable and did not display any further arrhythmias. He was extubated 26 hours later and amiodarone was continued. Transthoracic echocardiography and cardiac magnetic resonance imaging were performed, which returned normal results. His ECG had also returned to normal sinus rhythm (Fig 4). The herbal broth tested positive for aconitine upon toxicology screening. Other investigations during the hospital stay did not reveal any electrolyte abnormality and the toxicology screening did not reveal the use of any other illicit drugs or medications. The patient continued to improve and was discharged seven days later. At follow-up approximately one month later, he was doing well and reported no shortness of breath, chest pain or palpitations.

The case was referred to the government agency for pharmacovigilance, which examined the samples of herbs and the prescription together with the toxicology service. It was found that the patient had mistakenly copied down a dose of chuanwu and caowu (aconite root) that was ten times the safe dose.

\section{DISCUSSION}

Chuanwu and caowu are aconite root tubers used in traditional Chinese medicine for their analgesic and anti-inflammatory functions. They contain large amount of the alkaloid aconitine, mesaconitine and hypaconitine. (1) The estimated lethal dose of pure aconitine can be as low as $2 \mathrm{mg}$. ${ }^{(1)}$ The recommended dose of processed aconite roots has been lowered to 1.5-3 g; toxicity has been reported from consumption of 7-11 g of aconite roots. ${ }^{(2)}$ The roots must first be washed and soaked in water or saturated lime water. They are then boiled to reduce the toxic alkaloid content. ${ }^{(2)}$ By processing it in this manner, a less toxic form of aconitine alkaloid is formed. ${ }^{(3)}$ Our patient had a written prescription that required $60 \mathrm{~g}$ of aconite root as he had wrongly copied a recipe that was obtained from the Internet.

Aconitine and similar alkaloids bind to the open state of voltage-sensitive sodium channels in excitable tissue, such as the myocardium, nerves and muscles. Aconitine binds to the sodium channel with high affinity and causes the channel to remain in an active state. This results in continuous depolarisation of the 


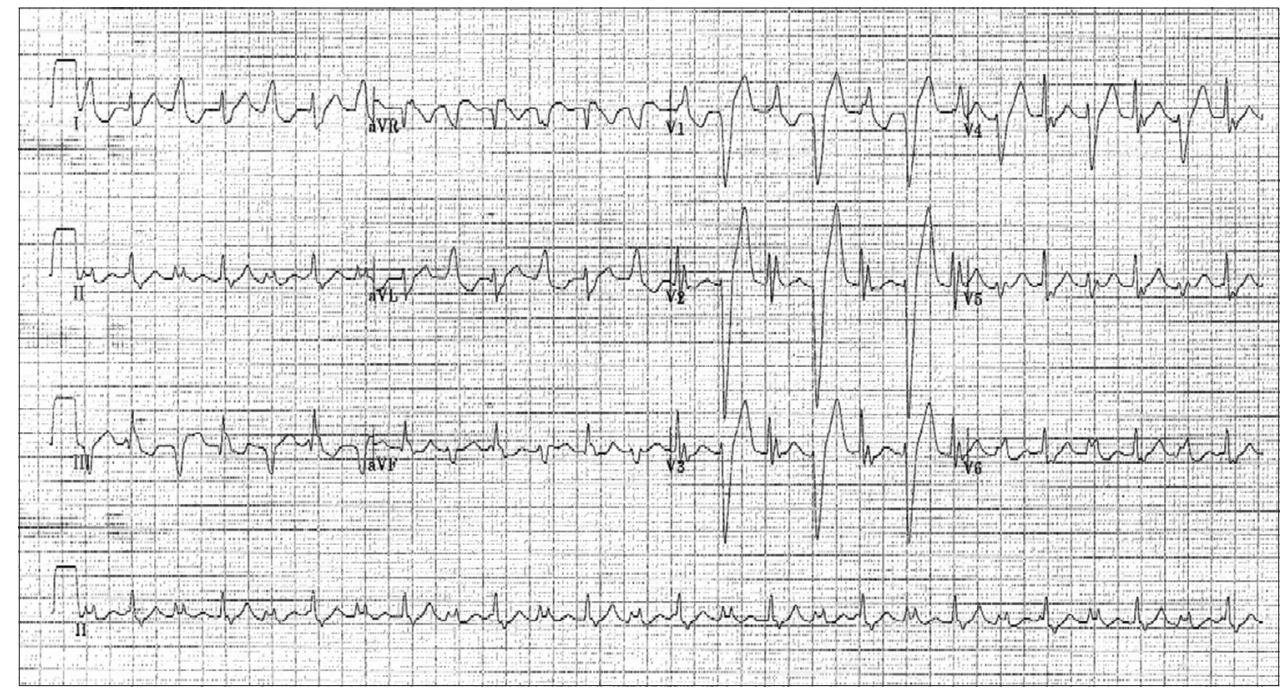

Fig. 3 ECG shows bidirectional ventricular tachycardia.

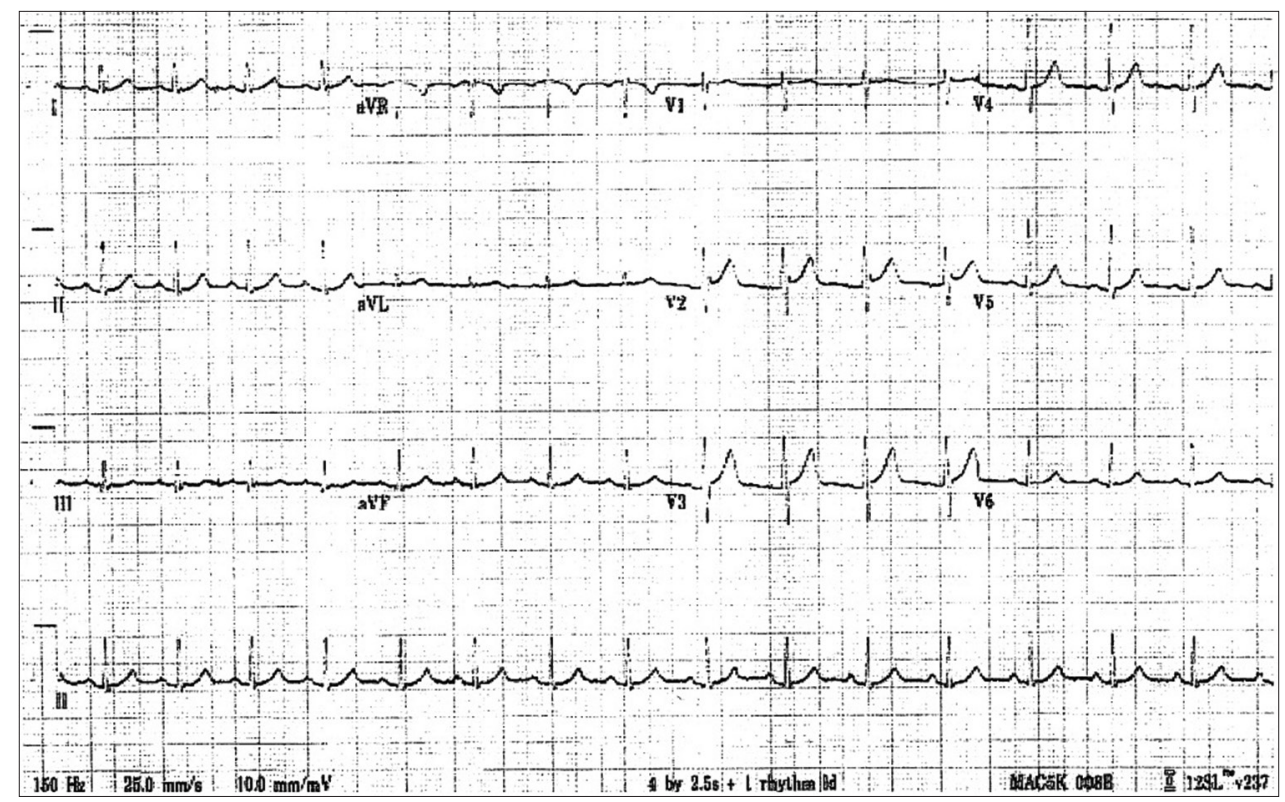

Fig. 4 ECG shows normal sinus rhythm.

sodium channels, which become refractory to excitation. ${ }^{(1)}$ This continuous state of depolarisation results in both peripheral and central actions. With oral ingestion of aconitine, symptoms of toxicity can appear as soon as 15 minutes to two hours after ingestion. ${ }^{(4)}$ The toxic effects in humans include gastrointestinal symptoms such as nausea, vomiting, diarrhoea and abdominal pain. Neurological features that include paraesthesia, numbness of face and muscle weakness are commonly present. ${ }^{(4)}$ Cardiovascular complications including hypotension, palpitations, ventricular ectopics, ventricular arrhythmias and death may ensue. ${ }^{(5)}$

One of the ventricular arrhythmias that can present in aconite poisoning is bidirectional VT. ${ }^{(6,7)}$ This is a rare ventricular dysrhythmia with beat-to-beat alternation of the frontal QRS axis. Bidirectional VT is a hallmark of severe digitalis toxicity and can also occur in cases of catecholaminergic polymorphic ventricular tachycardia. ${ }^{(8)}$ In patients presenting with this form of dysrhythmia from herbal poisoning, it is important to exclude digitalis toxicity as it can be treated with a digitalis antitoxin. In our patient, serum digoxin levels were undetectable.

Diagnosis of aconite poisoning usually depends on clinical signs and symptoms. Identification of the herb or the written prescription of aconite roots can also be useful. Tests for aconitine in herbal broth and biological fluid would help confirm the diagnosis. Our patient had relatively rapid onset of neurological manifestation and cardiotoxicity, although there was no significant gastrointestinal manifestation. He developed the typical electrographic changes of frequent PVCs and bidirectional VT. The diagnosis in this case was aided by the written prescription and identification of aconitine in the herbal broth.

There is no antidote for aconitine poisoning and the only treatment at present is supportive care. Ventricular arrhythmias, as in our case, are often refractory to cardioversion and antiarrhythmic drugs. First-line treatments that have been proven to be useful are the use of amiodarone and flecainide. ${ }^{(1)}$ For patients who are in cardiogenic shock and ventricular arrhythmias 
that do not resolve, a cardiopulmonary bypass may be necessary. The half-life of aconitine has been reported to be about three hours ${ }^{(4)}$ and with good supportive management, including extracorporeal support, the survival rate of the patient can be improved.

There have been several cases of overdose recorded in the literature. ${ }^{(1,2)}$ In some cases, patients have eaten the root in the wild believing that it was an edible plant. This resulted in severe toxicity requiring a percutaneous cardiopulmonary bypass and a prolonged hospital course. Another case was that of a 17-yearold man who took an intentional overdose of Aconitum napellus. It resulted in cardiac arrhythmias that required 30 shocks and antiarrythmic drugs, followed by cardiopulmonary support. The patient was hospitalised for 19 days but was discharged with no permanent damage. ${ }^{(9)}$ Cases that have been documented in the literature vary based on age, sex, treatment use, symptoms and ECG findings. In our patient, amiodarone was started early. Nevertheless, he progressed to ventricular fibrillation and cardiovascular collapse. The patient was refractory to medications and cardioversion, and required brief cardiopulmonary support. He did not, however, require bypass support and improved quite rapidly without any neurological or cardiac findings.

Patients need to be vigilant when consuming medications that contain aconitine. A study conducted in Hong Kong stated that there were four main reasons for overdose: (a) prescription error; (b) inadequately prepared herbs; (c) accidental dispensing; and (d) dispensary error. ${ }^{(10)}$ Patients should inform the dispensary of their medical problems and all medications they are taking to minimise the risk of interactions. There is a good argument for creating a standardised structure to train those who prepare and dispense the herb. As each herb has a different potency depending on where it is acquired, the process of growing the herb, then transporting, processing and dispensing it should be standardised. There is no such structure at present. As in modern medicine, there should also be a clear method of differentiating herbs that have similar names so that they are not mistaken for one another. Until processes for traditional medicine are more standardised, healthcare workers need to be constantly vigilant to look out for toxic overdose situations. In addition, there is a danger of self-medication from treatment recipes obtained from the Internet, especially when the information is not from a reputable source. The public should be educated to be cautious when self-medicating based on less reputable sources.

\section{ACKNOWLEDGEMENT}

We would like to thank Dr Yea Kok Chin for his help in the identification of the aconite roots in the prescription and from the samples brought by the family.

\section{REFERENCES}

1. Chan TY. Aconite poisoning. Clin Toxicol (Phila) 2009; 47:279-85.

2. Lin CC, Chan TY, Deng JF. Clinical features and management of herbinduced aconitine poisoning. Ann Emerg Med 2004; 43:574-9.

3. Jaiswal $\mathrm{Y}$, Liang Z, Yong $\mathrm{P}$, Chen $\mathrm{H}$, Zhao Z. A comparative study on the traditional Indian Shodhana and Chinese processing methods for aconite roots by characterization and determination of the major components. Chem Cent J 2013; 7:169.

4. Moritz F, Compagnon P, Kaliszczak IG, et al. Severe acute poisoning with homemade Aconitum napellus capsules: toxicokinetic and clinical data. Clin Toxicol (Phila) 2005; 43:873-6.

5. Fujita $Y$, Terui $K$, Fujita $M$, et al. Five cases of aconite poisoning: toxicokinetics of aconitines. J Anal Toxicol 2007; 31:132-7.

6. Yeih DF, Chiang FT, Huang SK. Successful treatment of aconitine induced life threatening ventricular tachyarrhythmia with amiodarone. Heart 2000; 84:E8.

7. Smith SW, Shah RR, Hunt JL, Herzog CA. Bidirectional ventricular tachycardia resulting from herbal aconite poisoning. Ann Emerg Med 2005; 45:100-1.

8. Richter S, Brugada P. Bidirectional ventricular tachycardia. J Am Coll Cardiol 2009; 54:1189.

9. Niinuma $\mathrm{H}$, Aoki $\mathrm{H}$, Suzuki $\mathrm{T}$, et al. Two survival cases of severe aconite poisoning by percutaneous cardiopulmonary support system and cardiopulmonary bypass for fatal arrhythmia: a case report. Internet J Emerg Intensive Care Med 2003; 6:47.

10. Chen SP, Ng SW, Poon WT, et al. Aconite poisoning over 5 years: a case series in Hong Kong and lessons towards herbal safety. Drug Saf 2012; 35:575-87. 